\title{
Tunable magnon-magnon coupling in synthetic antiferromagnets
}

\author{
A. Sud,,${ }^{1,{ }^{*}}$ C. W. Zollitsch $\odot,{ }^{1}$ A. Kamimaki, ${ }^{2,3}$ T. Dion $\odot,{ }^{1}$ S. Khan, ${ }^{1}$ S. Iihama, ${ }^{4,5}$ S. Mizukami,,${ }^{3,5,6}$ and H. Kurebayashi ${ }^{1, \dagger}$ \\ ${ }^{1}$ London Centre for Nanotechnology, University College London, London WC1H OAH, United Kingdom \\ ${ }^{2}$ Department of Applied Physics, Tohoku University, Aoba 6-6-05, Sendai 980-8579, Japan \\ ${ }^{3}$ WPI Advanced Institude for Materials Research, Tohoku University, 2-1-1, Katahira, Sendai 980-8577, Japan \\ ${ }^{4}$ Frontier Research Institute for Interdisciplinary Sciences, Tohoku University, Sendai 980-8578, Japan \\ ${ }^{5}$ Center for Spintronics Research Network, Tohoku University, Sendai 980-8577, Japan \\ ${ }^{6}$ Center for Science and Innovation in Spintronics, Tohoku University, Sendai 980-8577, Japan
}

(Received 1 June 2020; revised 3 August 2020; accepted 4 August 2020; published 10 September 2020)

\begin{abstract}
In this work, we study magnon-magnon coupling in synthetic antiferromagnets (SyAFs) using microwave spectroscopy at room temperature. Two distinct spin-wave modes are clearly observed and are hybridized at degeneracy points. We provide a phenomenological model that captures the coupling phenomena and experimentally demonstrate that the coupling strength is controlled by the out-of-plane tilt angle as well as the interlayer exchange field. We numerically show that a spin-current mediated damping in SyAFs plays a role in influencing the coupling strength.
\end{abstract}

DOI: 10.1103/PhysRevB.102.100403

Generating new spin-wave states can be an enabling role for developing future spintronic/magnonic devices [1]. While individual spin-wave modes can be tailored by changing material parameters of host magnets, a novel approach of creating new spin-wave states is to couple two modes coherently by tuning them into resonance, where physical parameters of the coupled modes can also be modified. Although the coupling phenomena could be phenomenologically explained by a classical coupled-oscillator picture in general, microscopic descriptions of this type of hybridization are rich, offering novel functionalities of state control and energy/information transfer. For example, strong coupling of light-matter interaction is envisaged to offer fast and protected quantum information processing [2-4]. Within this expanding research domain, strong coupling between microwave photons and collective spins in magnetically ordered systems has been extensively studied in recent years [5-8].

Magnon-magnon coupling has an advantage over the lightmatter interaction, in terms of coupling strength. The coupling strength of light-matter interactions is sometimes significantly reduced by a lack of spatial mode overlapping of the two, and so scientists have made considerable efforts to achieve large coupling strength by designing optimum geometries for efficient mode-volume overlapping $[9,10]$. On the other hand, magnon-magnon interaction does not suffer from this since two modes normally reside within the same host media, providing mode overlapping of $100 \%$ or close to. While magnon-magnon coupling has been studied in single magnets [11-14] and magnetic bilayers [15-17], magnon-magnon interaction in highly tunable material systems could offer unexplored parameter spaces on which to tailor the coupling

\footnotetext{
*aakanksha.sud.17@ucl.ac.uk

†h.kurebayashi@ucl.ac.uk
}

phenomena. Here, we focus on synthetic antiferromagnets (SyAFs) as a host of magnon-magnon coupling and report clear hybridization of two distinct SyAF modes arising from interlayer exchange coupling between two magnetic layers. We provide a full phenomenological model for the mode coupling, magnetic relaxation, and coupling strength as a function of different material parameters for SyAF modes. Aided by these derived relationships, we demonstrate that the interlayer exchange field strength, which can be controlled by sample growth, allows the engineering of the coupling strength. We further numerically show that the spin-current mediated damping plays a role in influencing the coupling strength. Our demonstration and full details of the magnonmagnon coupling phenomena in SyAFs will act as a springboard for further research along this avenue [18].

Low-energy spin-wave modes in synthetic antiferromagnets in their canted regime are acoustic and optical modes [19-21] where two coupled moments precess in-phase (acoustic) and out-of-phase (optical) as shown in Fig. 1(a). These modes have been studied and discussed already around the 1990s, e.g., by Grunberg et al. using Brilloin light scattering [22] and Zhang et al. by microwave cavity experiments [23]. The acoustic (optical) mode is excited by perpendicular (parallel) configuration between microwave and applied magnetic fields. There are a number of reports in which these two modes in different SyAFs have been studied in great detail [24-28]. For example, mutual spin pumping within the coupled moments has been proposed [29-31] and experimentally demonstrated [32-35]. Both optical and acoustic mode frequencies as a function of the middle layer that influences the interlayer exchange coupling strength have been studied and reported earlier [35,36]. Since the resonant frequency of two modes shows different magnetic field dependence (as discussed more later), we can find the degeneracy point of the two modes by tuning experimental conditions. When the two moments are 
(a)

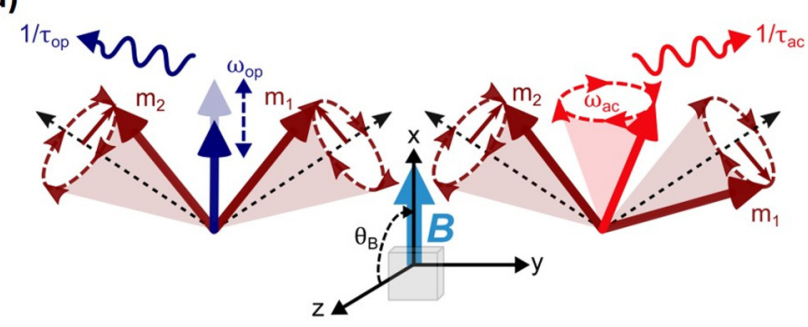

(b)

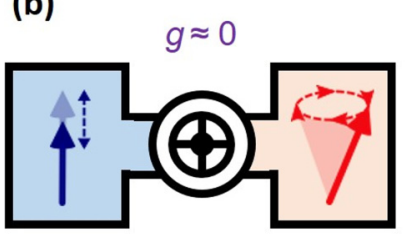

small $B_{\mathrm{ex}}$ or $\theta_{\mathrm{B}}=\pi / 2$

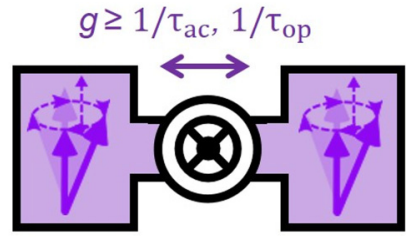

large $B_{\mathrm{ex}}$ and $\theta_{\mathrm{B}} \ll \pi / 2$
FIG. 1. (a) Schematic of acoustic and optical modes in SyAFs. Two moments $\left(m_{1}\right.$ and $\left.m_{2}\right)$ are coupled antiferromagnetically and canted at equilibrium. Under microwave irradiation, they precess in-phase (acoustic mode) and out-of-phase (optical mode) at different angular frequencies $\omega_{\mathrm{ac}}$ and $\omega_{\mathrm{op}}$, respectively. We define $\theta_{\mathrm{B}}$ as in the figure, where the $z$ axis is the film growth direction. (b) Schematics of the magnon-magnon coupling phenomena with the optical and acoustic modes. When the exchange field $\left(B_{\text {ex }}\right)$ is small or two moments are within the film plane, the coupling strength $(g)$ is zero, so the two modes do not couple. We can valve the coupling strength by tuning $B_{\mathrm{ex}}$ and $\theta_{\mathrm{B}}$ and achieve strong magnon-magnon hybridization, as shown in the right panel.

canted within the plane, the motion of the optical and acoustic modes can be decoupled [14], meaning that the two modes are not allowed to hybridize. This restriction can be lifted when we tilt the moments towards the out-of-plane direction and we will be able to hybridize them [see Fig. 1(b) for schematic understanding]. The strength of hybridization is defined by $g$ which represents a rate of energy transfer between the two modes. When this rate is fast, compared to mode dissipation rates of individual modes, we expect well-defined coupled modes before the excited states are relaxed. Control of the coupling strength in situ and ex situ will be potentially useful to a scheme of reconfigurable energy and information transfer using coherent coupling.

The SyAF stacked films used in this study were prepared by magnetron cosputtering at a base pressure of $1 \times 10^{-7} \mathrm{~Pa}$. The films were grown on a $\mathrm{Si}$ oxide substrate with the stacking pattern of $\mathrm{Ta}(3 \mathrm{~nm}) / \mathrm{CoFeB}(3 \mathrm{~nm}) / \mathrm{Ru}(\mathrm{t} \mathrm{nm}) / \mathrm{CoFeB}(3$ $\mathrm{nm}) / \mathrm{Ta}(3 \mathrm{~nm})$ where $\mathrm{Ru}$ thickness was varied to tune the interlayer exchange coupling [26]. Vibrating sample magnetometer was used to characterize the static magnetic properties (see Supplemental Material [37]). These sample chips were placed on a coplanar waveguide board to perform broadband spin dynamics characterization. For each measurement, we fixed the frequency and swept a dc external magnetic field with an ac modulation component at $12 \mathrm{~Hz}$. Figure 2(a) shows typical measurement curves for two field directions $\left(B_{\|}\right.$and $B_{\perp}$ ) defined by the figure inset. We carried out systematic experiments for a wide range of frequency $(5-20 \mathrm{GHz})$ as well as field angle to study mode hybridization and linewidth
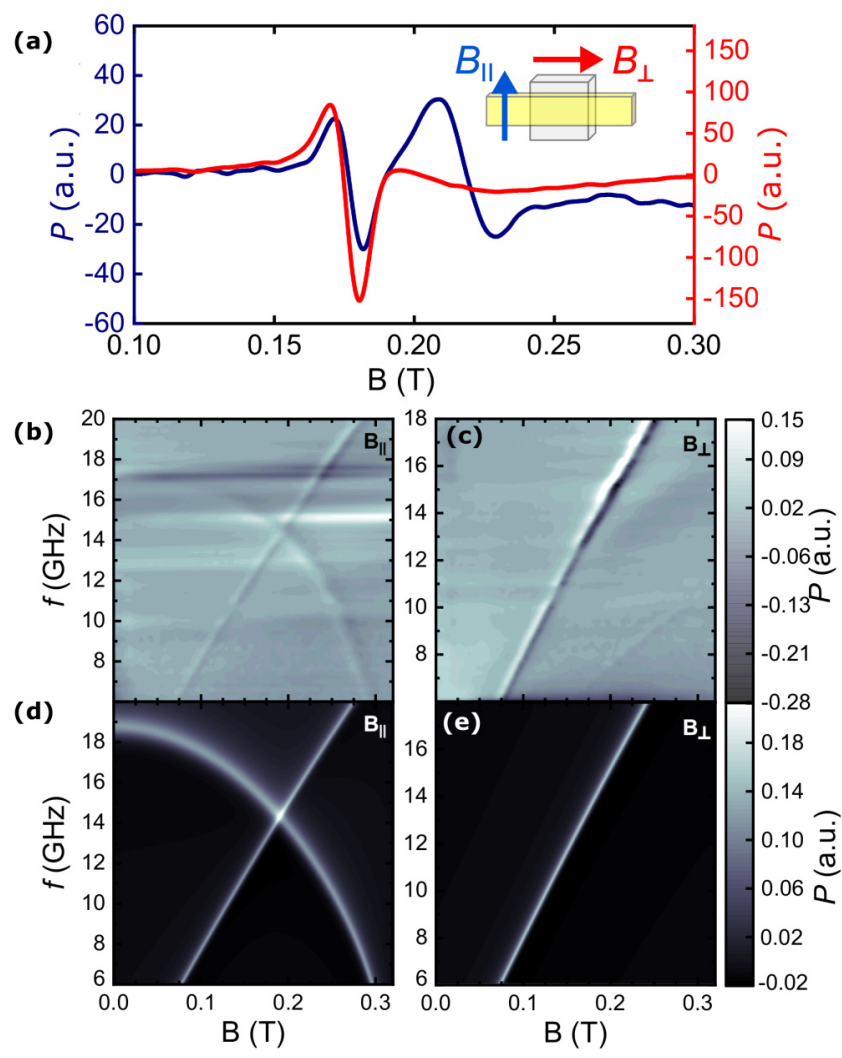

FIG. 2. (a) Microwave absorption spectrum for $\theta_{\mathrm{B}}=90^{\circ}$, measured at $13.4 \mathrm{GHz}$. Two magnetic field directions $\left(B_{\|}\right.$and $\left.B_{\perp}\right)$ are defined as per the inset. Microwave transmission spectrum as a function of frequency and applied field for two configurations of applied magnetic fields (b) $B_{\|}$and (c) $B_{\perp}$ for $\theta_{\mathrm{B}}=90^{\circ}$. (d), (e) Theoretical results for the same experimental conditions as (b) and (c), respectively.

evolution of SyAFs. To extract the peak position and linewidth, we used derivative Lorentzian functions [38]. Figures 2(b) and 2(c) show two-dimensional color plots of microwave absorption as a function of microwave frequency and magnetic field. We can clearly identify two modes in Fig. 2(b) for the $B_{\|}$condition whereas only one in Fig. 2(c) for $B_{\perp}$. This is because for $B_{\|}$, both modes can be excited since microwave rf fields have components both parallel and perpendicular to $B_{\|}$due to their spatial distribution above the waveguide. For $B_{\perp}$ measurements, the microwave magnetic field only possesses components perpendicular to $B_{\perp}$, hence only exciting the acoustic mode. In order to analyze these results quantitatively, we solve a coupled Landau-Lifshtitz-Gilbert (LLG) equation with small angle approximation [14,39-41] (see Supplemental Material [37] for more details) and find the resonance condition of the two modes as

$$
\begin{gathered}
\omega_{\mathrm{ac}}=\gamma B_{0} \sqrt{\left(1+\frac{B_{\mathrm{s}}}{2 B_{\mathrm{ex}}}\right)}, \\
\omega_{\mathrm{op}}=\gamma \sqrt{2 B_{\mathrm{ex}} B_{\mathrm{s}}\left[1-\left(\frac{B_{0}}{2 B_{\mathrm{ex}}}\right)^{2}\right]} .
\end{gathered}
$$




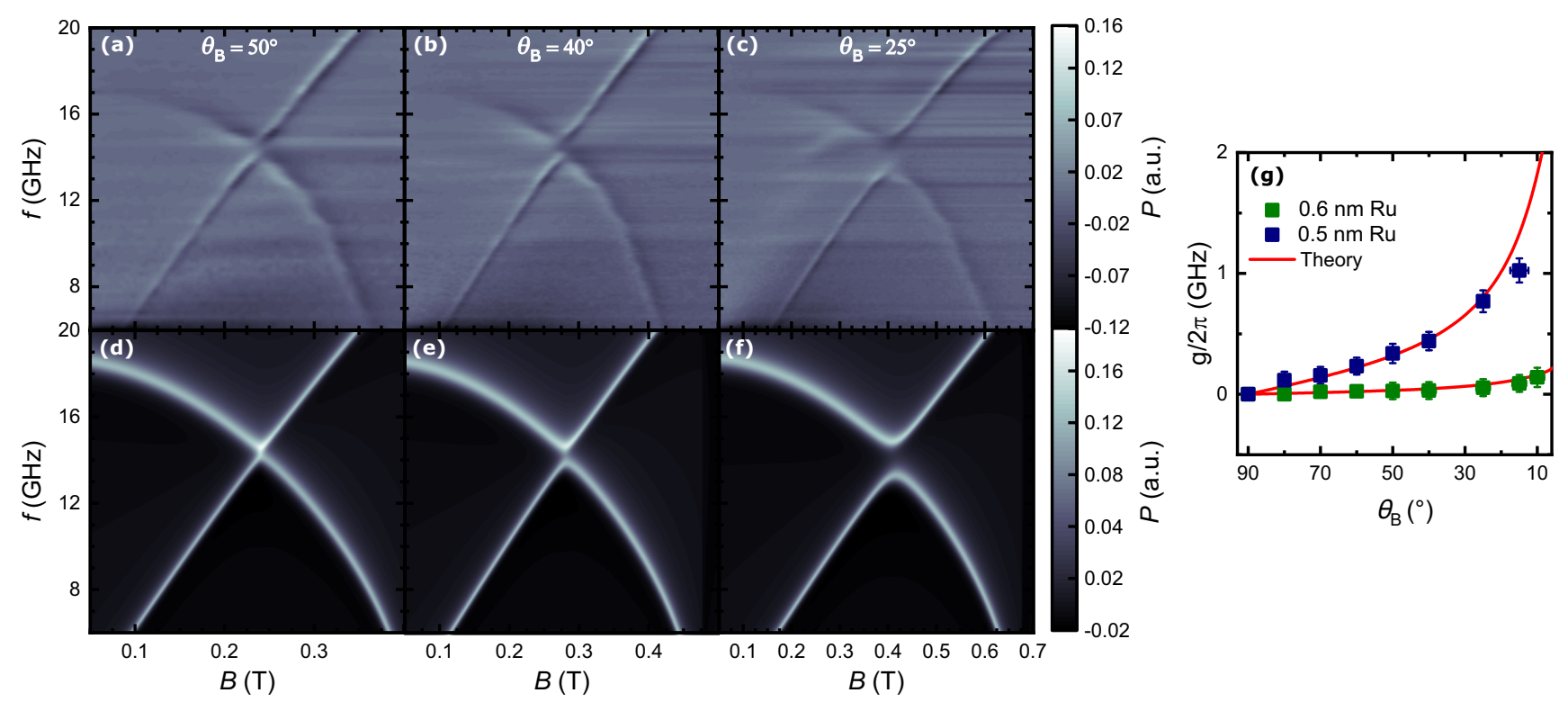

FIG. 3. (a)-(c) Microwave transmission as a function of frequency and applied field for different $\theta_{\mathrm{B}}$. The avoided crossing starts to appear and the frequency gap increases as $\theta_{\mathrm{B}}$ is decreased. (d)-(f) Simulation results for the same experimental condition as (a)-(c), respectively. (g) The coupling strength $g / 2 \pi$ as a function of $\theta_{\mathrm{B}}$. We plot results from two samples with the Ru thickness of $0.5 \mathrm{and} 0.6 \mathrm{~nm}$. The 0.5 -nm sample shows sizable $g / 2 \pi$, compared to much smaller $g / 2 \pi$ for $0.6 \mathrm{~nm}$. The red curves are produced by Eq. (3) in the main text.

Here, $B_{\mathrm{ex}}, B_{\mathrm{s}}, B_{0}$, and $\gamma$ are the exchange field, the demagnetization magnetization, the resonance field and the gyromagnetic ratio, respectively. We found that our best fits produce $B_{\mathrm{ex}}, B_{\mathrm{s}}$, and $\gamma / 2 \pi$ to be $0.14 \mathrm{~T}, 1.5 \mathrm{~T}$, and $29 \mathrm{GHz} / \mathrm{T}$, respectively. Resonance frequencies predicted by Eqs. (1) and (2) can reproduce our experimental results very well as shown in Figs. 2(d) and 2(e), strongly supporting that we can experimentally observe and study the coupled SyAF modes. Since the frequency of the two modes show different magnetic field dependencies, it is possible to study mode coupling of the two by tuning the mode frequencies. In Fig. 2(b), we observe a clear crossing of the two modes at $B_{0} \approx 0.2 \mathrm{~T}$. This "crossing" means that the two modes are not able to hybridize due to mode symmetry [14]. We can break this symmetry by tilting the moment towards the out-of-plane direction. We therefore repeated similar experiments for $\theta_{\mathrm{B}} \neq 90^{\circ}$ as shown in Figs. 3(a)-3(c). The two modes start to show an avoided crossing as $\theta_{\mathrm{B}}$ is decreased, indicating mode hybridization which can be quantitatively discussed by using the coupling strength $g / 2 \pi$, the half of the minimum frequency gap. We plot the $\theta_{\mathrm{B}}$ dependence of $g / 2 \pi$ in Fig. $3(\mathrm{~g})$ where $g / 2 \pi$ grows with the out-of-plane component, with the highest value exceeding $1 \mathrm{GHz}$.

We describe the magnon-magnon coupling phenomena in SyAFs by a $2 \times 2$ matrix eigenvalue problem derived from the coupled LLG equations with mutual spin pumping terms [31] (see Supplemental Material [37] for detailed derivation):

$$
\left[\begin{array}{cc}
\omega^{2}-\omega_{\mathrm{op}}^{2}+i\left(v_{\mathrm{o} 1}+v_{\mathrm{o} 2}\right) \omega & \left(i \omega-v_{\mathrm{o} 1} \gamma B_{s}\right) \eta m_{z 0} \\
\left(-i \omega+v_{\mathrm{a} 2} \gamma B_{s}\right) \eta m_{z} & \omega^{2}-\omega_{\mathrm{ac}}^{2}+i\left(v_{\mathrm{a} 1}+v_{\mathrm{a} 2}\right) \omega
\end{array}\right] .
$$

Here, $\eta=2 B_{\text {ex }} / B_{\mathrm{s}}, m_{z 0}=B_{0} \cos \theta_{\mathrm{B}} /\left(B_{\mathrm{s}}+2 B_{\mathrm{ex}}\right), v_{\mathrm{o} 1}=\left(\alpha_{0}+\right.$ $\left.\alpha_{\mathrm{sp}}\right)\left(1-m_{z 0}^{2}\right)-\alpha_{\mathrm{sp}}\left\{1-m_{z 0}^{2}-\left(B_{0}^{2} \sin ^{2} \theta_{\mathrm{B}} / 4 B_{\mathrm{ex}}^{2}\right)\right\}\left(m_{z 0}^{2} / m^{2}\right)$, $v_{o 2}=\alpha_{0} \eta\left(1-B_{0}^{2} \sin ^{2} \theta_{\mathrm{B}} / 4 B_{\mathrm{ex}}^{2}\right), \quad v_{\mathrm{a} 1}=\alpha_{0} \eta\left(m_{z 0}^{2}+B_{0}^{2} \sin ^{2} \theta_{\mathrm{B}} /\right.$ $\left.4 B_{\mathrm{ex}}^{2}\right)$, and $v_{a 2}=\alpha_{0}(\eta+1)\left(1-m_{z 0}^{2}\right)$, respectively, with $\alpha_{0}$ and $\alpha_{\mathrm{sp}}$ being the standard Gilbert damping constant and one arising from mutual spin pumping between the two magnetic layers. The real part of the eigenvalues gives the resonance frequencies and the imaginary part represents the loss rates of the two modes. We numerically solved the eigenvalue problem with parameters described above and found that the coupled equations can model our experimental observation well for each experimental set, such as Figs. 3(d)-3(f) reproducing corresponding experimental results. We simplified the $2 \times 2$ matrix by neglecting the damping terms to calculate the eigenvalues and found an analytical expression for the coupling strength as (see derivation in Supplemental Material [37])

$$
g=\frac{\gamma B_{\mathrm{ex}} B_{0}}{2 B_{\mathrm{s}}+4 B_{\mathrm{ex}}} \cos \theta_{\mathrm{B}}
$$

This correctly captures our experimental observation as $g / 2 \pi$ grows with decreasing $\theta_{\mathrm{B}}$. The red curve in Fig. $3(\mathrm{~g})$ is calculated by this equation and there is quantitative agreement between experiments and theory, despite marginal deviation at small $\theta_{\mathrm{B}}$. To further attest the validity of this equation for our experiments, we performed similar measurements on a SyAF sample having the Ru thickness of $0.6 \mathrm{~nm}$ since Eq. (3) suggests that the coupling strength can be tuned by $B_{\mathrm{ex}}$. For this sample, we found that $B_{\mathrm{ex}}$ is decreased to $30 \mathrm{mT}$ due to a weaker interlayer coupling and accordingly, as expected, we observed a significant decrease of $g / 2 \pi$ as summarized in Fig. $3(\mathrm{~g})$. These results show the tunability of the mode coupling strength in SyAFs by both thin-film growth engineering (ex situ) as well as out-of-plane tilt angle (in situ).

Next we focus on the relaxation of the SyAF modes. Figures 4(a) and 4(b) represent plots of the half width at half maximum (HWHM) linewidth $(\Delta B)$ extracted for individual sweeps for both modes. $\Delta B$ of the acoustic mode increases with increasing magnetic field, with a characteristic anormaly 

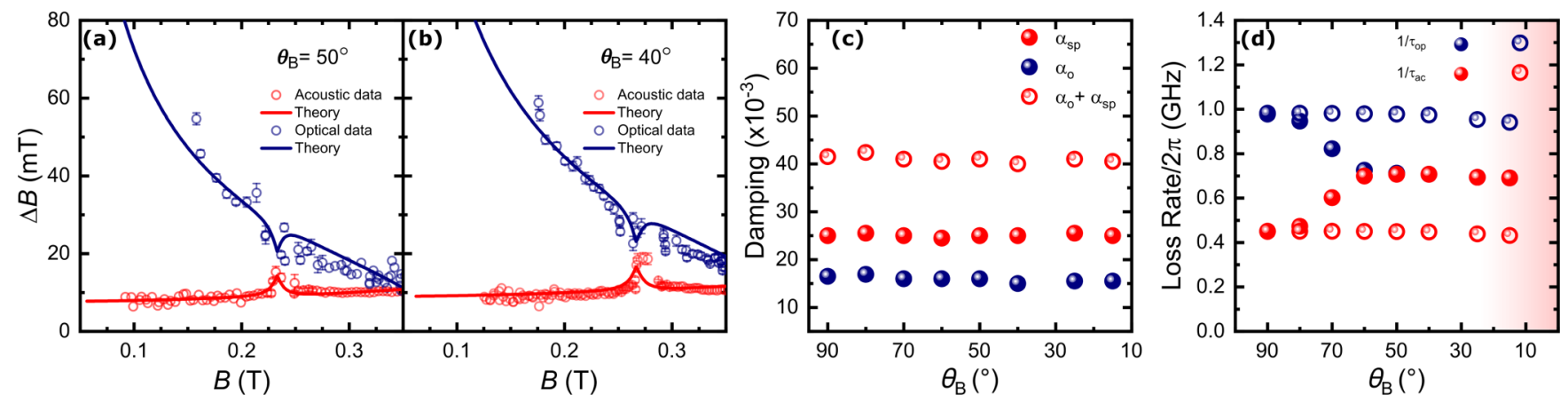

FIG. 4. (a)-(c) HWHM linewidth as a function of magnetic field for $\theta_{B}$ of (a) $50^{\circ}$ and (b) $40^{\circ}$. Solid lines represent results from the theoretical model discussed in the main text. (c) Extracted values of damping parameters. (d) Calculated loss rates of each mode at the crossing point as well as those of the hybridized modes.

around the field where the two modes hybridize. $\Delta B$ of the optical mode, however, shows a different magnetic field dependence as it decreases with increasing magnetic field. This is primarily due to the relationship of the magneticfield-domain linewidth and frequency-domain linewidth as given by

$$
\Delta B_{\mathrm{op}(\mathrm{ac})}=\left|\frac{d \omega_{\mathrm{op}(\mathrm{ac})}}{d B}\right|^{-1} \frac{1}{\tau_{\mathrm{op}(\mathrm{ac})}} .
$$

When the resonance field is low, $\left|d \omega_{\mathrm{op}} / d B\right|$ becomes small, which can extrinsically enhance the observed $\Delta B$ in our experiments. In order to extract material-specific parameters such as $\alpha_{0}$ from our data, we solved the eigenvalue problem and compared the imaginary part with experimental results. We found that the linewidth calculated from the imaginary part models excellently for our experiments as shown in Figs. 4(a) and 4(b). Extracted $\alpha_{0}$ and $\alpha_{\mathrm{sp}}$ for different $\theta_{\mathrm{B}}$ are plotted in Fig. 4(c). We can confirm that there is a sizable spin pumping component for every angle we measured, consistent with previous reports $[26,31,34,42]$. The Ru thickness is much shorter than its spin diffusion length of $14 \mathrm{~nm}$ [43]. As a result, when two ferromagnets are precessing in-phase, according to spin pumping theory [44], spin currents flowing out of the two are canceled out hence developing zero time-dependent spin accumulation in the Ru layer. However, when two moments precess out of phase, the emitted spin currents no longer cancel out, leading to the spin accumulation which induces an additional damping mechanism for the optical mode. In our experiments, we observe that both $\alpha_{0}$ and $\alpha_{\mathrm{sp}}$ are independent of $\theta_{\mathrm{B}}$, which can be understood that the Gilbert damping components are a material parameter, independent of experimental conditions. Note here that the canted angle has been already taken into account in the expressions.

An interesting observation is that the experimentally deduced $\Delta B$ for both modes also show "attraction" around the avoided crossing points. This demonstrates that magnetic relaxation can be modified by mode coupling phenomena. In the crossing regime, two modes are no longer pure acoustic or optical and therefore it is not possible to use the ac spin pumping picture associated with the phase difference between two moments. Rather, a simple phenomenological picture of hybridized energy losses would be a better one. When two modes with different loss rates start to couple coherently, their loss rates also start to merge together [45]. This is because the energy transfer mixes the two loss rates since the high(low-) loss mode becomes the low- (high-) loss mode as a function of time. We are able to observe this feature in our experiments. This loss rate hybridization is reproduced by our numerical simulations from the eigenvalue problem as shown in Figs. 4(a) and 4(b). This linewidth averaging is similar to those discussed in spin-photon coupling systems [35,46,47] as well as magnon-magnon coupling at $\mathrm{YIG} / \mathrm{NiFe}$ interfaces [17]. We went on to quantify the loss rates for both modes by using Eq. (4). First of all, we estimated the loss rate of individual modes at the avoided crossing point [open circles in Fig. 4(d)], by extrapolating from the values outside the coupling regime. Both show a very weak angular dependence, which can be understood that the damping [Fig. 4(c)] has no angular dependence with a subtle change of the modecrossing frequency when $\theta_{\mathrm{B}}$ is decreased. By contrast, loss rates for the hybridized modes [solid circles in Fig. 4(d)], estimated by our eigenvalue problem, exhibit clear attraction as the coupling strength is increased by changing $\theta_{\mathrm{B}}$. After $\theta_{\mathrm{B}}=60^{\circ}$, the loss rates of the two modes coalesce into a single number which is exactly the average of the two rates $1 / \tau_{\text {mix }}=(1 / 2)\left(1 / \tau_{\mathrm{ac}}+1 / \tau_{\mathrm{op}}\right)$ where $1 / \tau_{\text {mix }}$ is the loss rate of the hybridized states. Furthermore, through the course of our simulation study, we found that $\alpha_{\mathrm{sp}}$ can have an effect on $g$, suggesting that the magnon-magnon coupling is partially mediated by spin currents. We observe that for large $\alpha_{\mathrm{sp}}$ the coupling between the two modes can be completely suppressed (see Supplemental Material [37]). We highlight that this damping-mediated coupling control cannot be achieved by simply changing $\alpha_{0}$ in our system, something specific for the magnetic relaxation via spin pumping to the coupling and the energy exchange. Although it is not possible to control $\alpha_{\mathrm{sp}}$ in our experiments, it could act as an extra parameter to define the magnon-magnon coupling strength in SyAFs. Finally, we highlight that the highest $g / 2 \pi$ achieved $(1.0 \mathrm{GHz})$ outnumbers the loss rates of the individual modes, indicating that this magnon-magnon coupling starts to enter the strong-coupling regime in our experiments. Although our experiments are just at the onset of the strong-coupling regime, here we briefly discuss potential improvements and control of the coupling strength against the individual loss rates. Equation (3) can be simplified as $g / 2 \pi \propto B_{\text {ex }} / B_{\mathrm{s}}$, suggesting that a sample with a higher $B_{\text {ex }}$ as well as a smaller $B_{\mathrm{s}}$ shows a large coupling 
strength. Achieving similar coupling with low-damping materials could be another plausible path.

In summary, we experimentally show the magnon-magnon coupling in SyAF $\mathrm{CoFeB} / \mathrm{Ru} / \mathrm{CoFeB}$ multilayers. Clear magnon-magnon hybridization has been observed when the optical and acoustic modes are tuned into resonance. The magnon-magnon coupling strength has been controlled by bringing the moments into the out-of-plane direction, which breaks the orthogonality of the two modes. In addition, the interlayer exchange coupling is found to tune the coupling strength. The loss rate of two modes exhibits an averaging effect upon hybridization. Our eigenvalue problem approach serves to provide the analytical expression of the coupling strength as well as numerical explanations/predictions of the experimental data. We envisage that results in the present study will be transferable to other weakly coupled antiferromagnetic systems since the phenomenological descriptions of their spin-wave modes should be identical to our model developed.

Note added. We became aware that similar magnonmagnon coupling in synthetic antiferromagnets has been observed for finite wavelength spin-waves by Shiota et al. [51].

A.K. acknowledges the Graduate Program in Spintronics (GP-Spin) at Tohoku University. This work was supported in part by CSRN, CSIS, and UCL-Tohoku Strategic Partner Funds.
[1] A. V. Chumak, V. I. Vasyuchka, A. A. Serga, and B. Hillebrands, Nat. Phys. 11, 453 (2015).

[2] A. F. Kockum, A. Miranowicz, S. D. Liberato, S. Savasta, and F. Nori, Nat. Rev. Phys. 1, 19 (2019).

[3] Z.-L. Xiang, S. Ashhab, J. Q. You, and F. Nori, Rev. Mod. Phys. 85, 623 (2013).

[4] A. A. Clerk, K. W. Lehnert, P. Bertet, J. R. Petta, and Y. Nakamura, Nat. Phys. 16, 257 (2020).

[5] D. Lachance-Quirion, Y. Tabuchi, A.Gloppe, K. Usami, and Y. Nakamura, Appl. Phys. Express 12, 070101 (2019).

[6] M. Harder and C.-M. Hu, in Solid State Physics 69, edited by R. E. Camley and R. L. Stamp (Academic, Cambridge, 2018), pp. 47-121.

[7] H. Huebl, C. W. Zollitsch, J. Lotze, F. Hocke, M. Greifenstein, A. Marx, R. Gross, and S. T. B. Goennenwein, Phys. Rev. Lett. 111, 127003 (2013).

[8] X. Zhang, C. L. Zou, L. Jiang, and H. X. Tang, Phys. Rev. Lett. 113, 156401 (2014).

[9] C. Eichler, A. J. Sigillito, S. A. Lyon, and J. R. Petta, Phys. Rev. Lett. 118, 037701 (2017).

[10] G. Tosi, F. A. Mohiyaddin, H. Huebl, and A. Morello, AIP Adv. 4, 087122 (2014).

[11] B. A. Kalinikos and A. N. Slavin, J. Phys. C: Solid State Phys. 19, 7013 (1986).

[12] P. Grünberg, in Light Scattering from Spin Waves in Thin Films and Layered Magnetic Structures in Light Scattering in Solids V, edited by M. Cardona and G. Güntherodt (Springer, NewYork, 1989).

[13] L. Liensberger, A. Kamra, H. Maier-Flaig, S. Geprägs, A. Erb, S. T. B. Goennenwein, R. Gross, W. Belzig, H. Huebl, and M. Weiler, Phys. Rev. Lett. 123, 117204 (2019).

[14] D. MacNeill, J. T. Hou, D. R. Klein, P. Zhang, P. Jarillo-Herrero, and L. Liu, Phys. Rev. Lett. 123, 047204 (2019).

[15] J. Chen, C. Liu, T. Liu, Y. Xiao, K. Xia, G. E. W. Bauer, M. Wu, and H. Yu, Phys. Rev. Lett. 120, 217202 (2018).

[16] S. Klingler, V. Amin, S. Geprags, K. Ganzhorn, H. Maier-Flaig, M. Althammer, H. Huebl, R. Gross, R. D. McMichael, M. D. Stiles, S. T. B. Goennenwein, and M. Weiler, Phys. Rev. Lett. 120, 127201 (2018).

[17] Y. Li, W. Cao, V. P. Amin, Z. Zhang, J. Gibbons, J. Sklenar, J. Pearson, P. M. Haney, M. D. Stiles, W. E. Bailey, V. Novosad,
A. Hoffmann, and W. Zhang, Phys. Rev. Lett. 124, 117202 (2020).

[18] R. A. Duine, K.-J. Lee, S. S. P. Parkin, and M. D. Stiles, Nat. Phys. 14, 217 (2018).

[19] F. Keffer and C. Kittel, Phys. Rev. 85, 329 (1952).

[20] S. M. Rezende, A. Azevedo, and R. L. Rodríguez-Suárez, J. Appl. Phys. 126, 151101 (2019).

[21] J. J. Krebs, P. Lubitz, A. Chaiken, and G. A. Prinz, J. Appl. Phys. 67, 5920 (1990).

[22] P. Grunberg, R. Schreiber, Y. Pang, M. B. Brodsky, and H. Sowers, Phys. Rev. Lett. 57, 2442 (1986).

[23] Z. Zhang, L. Zhou, P. E. Wigen, and K. Ounadjela, Phys. Rev. B 50, 6094 (1994).

[24] A. Konovalenko, E. Lindgren, S. S. Cherepov, V. Korenivski, and D. C. Worledge, Phys. Rev. B 80, 144425 (2009).

[25] T. Seki, H. Tomita, A. A. Tulapurkar, M. Shiraishi, T. Shinjo, and Y. Suzuk, Appl. Phys. Lett. 94, 212505 (2009).

[26] A. Kamimaki, S. Iihama, T. Taniguchi, and S. Mizukami, Appl. Phys. Lett. 115, 132402 (2019).

[27] W. Wang, P. Li, C. Cao, F. Liu, R. Tang, G. Chai, and C. Jiang, Appl. Phys. Lett. 113, 042401 (2018).

[28] M. Ishibashi, Y. Shiota, T. Li, S. Funada, T. Moriyama, and T. Ono, Sci. Adv. 6, eaaz6931 (2020).

[29] T. Taniguchi and H. Imamura, Phys. Rev. B 76, 092402 (2007).

[30] S. Takahashi, Appl. Phys. Lett. 104, 052407 (2014).

[31] T. Chiba, G. E. W. Bauer, and S. Takahashi, Phys. Rev. B 92, 054407 (2015).

[32] A. A. Timopheev, Y. G. Pogorelov, S. Cardoso, P. P. Freitas, G. N. Kakazei, and N. A. Sobolev, Phys. Rev. B 89, 144410 (2014).

[33] K. Tanaka, T. Moriyama, M. Nagata, T. Seki, K. Takanashi, S. Takahashi, and T. Ono, Appl. Phys. Express 7, 063010 (2014).

[34] H. Yang, Y. Li, and W. E. Bailey, Appl. Phys. Lett. 108, 242404 (2016).

[35] S. Sorokin, R. A. Gallardo, C. Fowley, K. Lenz, A. Titova, G. Y. P. Atcheson, G. Dennehy, K. Rode, J. Fassbender, J. Lindner, and A. M. Deac, Phys. Rev. B 101, 144410 (2020).

[36] X. M. Liu, H. T. Nguyen, J. Ding, M. G. Cottam, and A. O. Adeyeye, Phys. Rev. B 90, 064428 (2014).

[37] See Supplemental Material at http://link.aps.org/supplemental/ 10.1103/PhysRevB.102.100403 for supporting data and analysis for this study, which includes Refs. [48-51]. 
[38] K. Rogdakis, A. Sud, M. Amado, C. M. Lee, L. McKenzie-Sell, K.-R. Jeon, M. Cubukcu, M. G. Blamire, J. W. A. Robinson, L. F. Cohen, and H. Kurebayashi, Phys. Rev. Mater. 3, 014406 (2019).

[39] P. K. Streit and G. E. Everett, Phys. Rev. B 21, 169 (1980).

[40] V. S. Mandel, V. D. Voronkov, and D. E. Gromzin, Zh. Eksp. Teor. Fiz. 63, 993 (1972) [Sov. Phys. JETP 36, 521 (1973)].

[41] A. Kamimaki, S. Iihama, K. Z. Suzuki, N. Yoshinaga, and S. Mizukami, Phys. Rev. Appl. 13, 044036 (2020).

[42] B. Heinrich, Y. Tserkovnyak, G. Woltersdorf, A. Brataas, R. Urban, and G. E. W. Bauer, Phys. Rev. Lett. 90, 187601 (2003).

[43] K. Eid, R. Fonck, M. A. Darwish, W. P. Pratt, Jr., and J. Bass, J. Appl. Phys. 91, 8102 (2002).
[44] Y. Tserkovnyak, A. Brataas, and G. E. W. Bauer, Phys. Rev. Lett. 88, 117601 (2002).

[45] H. J. Carmichael, R. J. Brecha, M. G. Raizen, H. J. Kimble, and P. R. Rice, Phys. Rev. A 40, 5516 (1989).

[46] M. Harder, L. Bai, P. Hyde, and C.-M. Hu, Phys. Rev. B 95, 214411 (2017).

[47] M. Harder, Y. Yang, B. M. Yao, C. H. Yu, J. W. Rao, Y. S. Gui, R. L. Stamps, and C.-M. Hu, Phys. Rev. Lett. 121, 137203 (2018).

[48] S. O. Demokritov, J. Phys. D: Appl. Phys. 31, 925 (1998).

[49] M. Belmeguenai, T. Martin, G. Woltersdorf, M. Maier, and G. Bayreuther, Phys. Rev. B 76, 104414 (2007).

[50] S. M. Rezende, C. Chesman, M. A. Lucena, A. Azevedo, F. M. De Aguiar, and S. S. P. Parkin, J. Appl. Phys. 84, 958 (1998).

[51] Y. Shiota, T. Taniguchi, M. Ishibashi, T. Moriyama, and T. Ono, Phys. Rev. Lett. 125, 017203 (2020). 